\title{
Fever and Infection Prevalence in Hematology-Oncology Hospitalized Patients: A Single-Center Retrospective Study
}

\author{
Inês Almeida Costaㅁ, Sérgio Chacim² ${ }^{2}$ José Mariz ${ }^{2}$ \\ ${ }^{1}$ Internal Medicine Department, Centro Hospitalar de São João, Porto, Portugal \\ ${ }^{2}$ Departmentof Hematology-Oncology Clinic, Instituto Português de Oncologia (IPO), Porto, Portugal \\ Email: ines.almeidacosta@gmail.com
}

How to cite this paper: Costa, I.A., Chacim, S. and Mariz, J. (2018) Fever and Infection Prevalence in Hematology-Oncology Hospitalized Patients: A Single-Center Retrospective Study. Journal of Cancer Therapy, 9, 231-241.

https://doi.org/10.4236/jct.2018.93020

Received: January 17, 2018

Accepted: March 11, 2018

Published: March 14, 2018

Copyright $\odot 2018$ by authors and Scientific Research Publishing Inc. This work is licensed under the Creative Commons Attribution International License (CC BY 4.0).

http://creativecommons.org/licenses/by/4.0/

\begin{abstract}
Fever is prevalent in hospitalized patients. In hematology-oncology patients, it is related with infection, a disease manifestation or a consequence of immunological dysfunction. This single-center retrospective study aims to determine fever prevalence, describe its causes and evaluate the impact of infection on mortality. We reviewed clinical records of 222 patients admitted to the hospital in three random months of 2015. Around 35\% of all hospitalizations were complicated by fever, $42.5 \%$ of unknown origin. $90.4 \%$ were under treatment. Around $81 \%$ did antibiotics, most commonly piperacillin/tazobactam, for around 11 days. $41 \%$ changed antibiotics due to fever persistence or microbiological results. The diagnostic yield of microbiological tests was $25 \%$; in $66.7 \%$, a Gram-negative microorganism was isolated. We didn't find an association between infection and mortality. We conclude that fever is common in our hematology-oncology clinic and that the team is trained to assume infection; empirical treatment is in accordance with international recommendations.
\end{abstract}

\section{Keywords}

Fever, Infection, Hematology-Oncology, Onco-Hematology, Neutropenia

\section{Introduction}

Fever as a classical sign of infection was first described by Celsius at year 30 before Christ. In endothermic animals, it happens at the expense of high metabolic rates, with an increment of $10 \%$ to $12.5 \%$ for each degree Celsius above normal [1]. This mechanism allows for a better immune system activation and inhibi- 
tion of bacteria and viruses replication [2]. A corollary of fever benefits is its preservation throughout the evolution of species [3].

Fever is a frequent finding in hospitalized patients. A study of 1987 about fever incidence, prevalence and causes in patients admitted to a municipal hospital of Atlanta, United States, documented $29 \%$ of febrile episodes. Proved or suspected infection comprised $74 \%$ of all cases, while the remaining $26 \%$ were caused by neurological diseases (mainly intracranial, subdural or subarachnoid hemorrhage), malignancy, cardiac and pulmonary diseases, drug-associated reactions, thrombophlebitis or blood/blood-derived products transfusion reactions [4].

Fever can affect as much as $80 \%$ of all hematology-oncology patients, particularly during neutropenia [5]. This high prevalence seems to be related with infection-a common complication of immunological dysfunction due to bone marrow invasion and/or cytotoxic treatments-, but fever might also be part of disease manifestations or a direct consequence of other drugs commonly used, such as high-dose cytarabin and amphotericin [6].

Few recent works studied the prevalence and causes of fever in hematology-oncology patients [7], and most of them focused on fever in neutropenic patients [6] [8] [9] [10]. We hypothesize that most febrile episodes in this population are secondary to infection. Since infection alone appears to worsen prognosis by raising mortality or by requiring chemotherapy adjustments or suspension [10], knowing fever prevalence in this group of patients is of great importance.

We conducted a retrospective study in a sample of hematology-oncology patients to determine overall prevalence of fever and its causes, with particular emphasis to infection. Secondary aims were to revise microbiological isolates and evaluate the impact of infection on mortality.

\section{Methods}

The Portuguese Oncology Institute (Instituto Português de Oncologia-IPO) of Porto is an oncology hospital located in the northern part of Portugal. The hematology-oncology clinic has around 600 new patients each year; besides outpatient facilities, it includes a ward with 12 positive pressure isolation bedrooms and a total of 20 beds.

This work was approved by the Ethics Committee of IPO-Porto. Since data was extracted preserving the confidentiality of each patient and there was no direct contact with patients, informed consent was waived.

We reviewed clinical records of all patients admitted to the clinic during April, September and November 2015. These three months were arbitrarily selected by a randomization tool available at [https://www.random.org]. Patients below 18 years-old, transferred from other hospitals or admitted to the hematology-oncology clinic but not staying physically at our ward were excluded. Data on demographics, admission diagnosis, hematological disease, type and response to treatment, prophylactic antimicrobials in use, medical devices, signs 
and symptoms, leukocyte and neutrophil count, findings in other diagnostic tests, microbiological products and isolates, antibiotics choice and need of revision, admission to intensive care unit (ICU), and outcomes at discharge and at 30 and 90 days was collected.

For purposes of homogeneity, we established the following propositions:

1) Each febrile episode was treated as a case.

2) A febrile episode was considered solved after 72 or 96 hours of apyrexia, depending on the type of patient (non-neutropenic or neutropenic, respectively) [4].

3) Fever was defined as tympanic temperature of $\geq 38.3^{\circ} \mathrm{C}$ for at least an hour.

4) Chemotherapy was considered active until three months after suspension.

5) Intensive chemotherapy was defined as that with "the highest potential of febrile neutropenia" [9]. Chemotherapy protocols for acute myeloid leukemia, acute lymphoid leukemia, Burkitt lymphomas and second lines for Hodgkin and non-Hodgkin's lymphoma were included in this definition.

6) Bacterial skin flora was assumed as probably infective if they were isolated in two different blood culture sets, collected in different timings.

We performed a descriptive analysis of all study variables, calculating means and standard deviation for continuous variables and proportions for categorical variables. Subgroup analysis was performed by chi-square test. All data was analyzed with $24^{\text {th }}$ edition of IBM Statistical Package for the Social Sciences ${ }^{\circledR}$. A $p$-value below 0.05 was considered statistically significant.

\section{Results}

There were 992 hospitalizations in the hematology-oncology clinic in 2015, with 222 happening in the three-month period defined. After excluding eight hospitalizations corresponding to patients transferred from other hospitals and 25 for being admitted to other wards, a total of 189 hospitalizations were considered for analysis. There were 73 febrile episodes, affecting 66 (34.9\%) hospitalizations and 54 different patients.

Sample is composed by 28 males $(51.9 \%)$; mean age was $59.3( \pm 12.6)$ years old.

\subsection{Febrile Episodes}

Among all 73 febrile episodes, 16 (21.9\%) were in patients with lymphoid leukemia, $14(19.2 \%)$ in patients with myeloid leukemia and $8(11 \%)$ in patients with aplastic anemia or myelodysplastic syndromes. The remaining episodes were in patients with mantle cell lymphoma, diffuse large B-cell lymphoma, multiple myeloma, Hodgkin lymphoma, peripheral T-cell lymphoma, myeloid sarcoma and Burkitt lymphoma.

Of all 73 febrile episodes, 66 (90.4\%) happened in patients under active treatment for their hematology-oncology disease, 39 (53.5\%) of which were intensive protocols. 7 (9.6\%) febrile episodes took place in patients under no treatment. 
Concerning disease status, 17 (23.3\%) febrile episodes occurred in patients with progressive disease, $13(17.8 \%)$ in complete remission, $8(11 \%)$ with stable disease and $6(8.2 \%)$ with recurrent disease. In 29 (39.7\%) of all 73 febrile episodes, disease status was unknown.

A majority of patients (57.5\% and 63\%, respectively) were doing prophylactic antibiotics (usually trimethoprim-sulphamethoxazole $960 \mathrm{mg}$ three times a week and acyclovir $400 \mathrm{mg}$ twice a day); prophylactic antifungal therapy (usually itraconazole) was prescribed in a modest percentage (27\%).

Around $52 \%$ of all febrile episodes took place in patients with double lumen $7 \mathrm{fr}$ central venous access (CVA).

In $42.5 \%$ of cases it was not possible to determine the cause of fever either clinically, from laboratory tests or by imaging; these cases were grouped as "fever of unknown origin". Bacteremias (20.5\%) and respiratory tract infections (16.4\%) were the second and third most common causes of fever.

\subsection{Microbiological Isolates}

There were $25 \%$ of microbiological isolates in the whole sample, $16.4 \%$ of which in blood cultures, $4.1 \%$ in urine cultures and $4.1 \%$ in more than one biological product (any combination of blood cultures, urine cultures or respiratory secretions).

Table 1 displays all microbiological isolates, with $66.7 \%$ being Gram-negative and 33\% Gram-positive.

\subsection{Antibiotics}

Antibiotics were empirically started in the majority of febrile episodes, with $43.8 \%$ beginning with piperacillin/tazobactam, $15.1 \%$ with meropenem and $21.9 \%$ with a combination of at least two drugs (Table 2).

In $41.1 \%$ of all cases antibiotics were changed because of persistent fever or to adjust to the isolated microorganism. This change was mostly intended to increase antibiotics spectrum, and was done by adding another antimicrobial (26\%) or by actually changing antibiotics to a different one (15.1\%). Antibiotics substitution due to persistent fever happened after a mean of $4.18(+/-4.1)$ days of raised temperature.

Each febrile episode lasted an average of $3.19(+/-4.63)$ days; antibiotics were done for an average of $11.14(+/-6.76)$ days.

\subsection{Outcome}

Two patients were admitted in the ICU unit.

The in-hospital mortality rate was $13.7 \%$; mortality rose to $28.8 \%$ and $34.3 \%$ when we analyzed outcomes at 30 and 90 days, respectively.

\section{Discussion}

Our study shows that almost $35 \%$ of all hospitalizations in a hematology-oncology clinic of a portuguese oncology hospital were complicated by fever. 
Table 1. Description of all microbiological isolates of blood samples drawn from patients with fever.

\begin{tabular}{ccc}
\hline & Number $(\mathrm{n})$ & Percentage (\%) \\
\hline Klebsiella pneumoniae & 4 & 22.2 \\
Escherichia coli & 3 & 16.7 \\
Enterobacter cloacae & 2 & 11.1 \\
Pseudomonas aeruginosa & 2 & 11.1 \\
Acinetobacter lwoffii & 1 & 5.55 \\
Enterococcus faecalis & 1 & 5.55 \\
Staph. aureus & 1 & 5.56 \\
Staph. epidermidis & 1 & 5.56 \\
Staph. haemolyticus & 1 & 5.56 \\
Streptococcus dysgalactiae & 1 & 5.56 \\
Streptococcus mitis & 1 & 5.56 \\
Total & 18 & 100.0 \\
\hline
\end{tabular}

Table 2. Description of antibiotics used in patients with fever due to infection.

\begin{tabular}{|c|c|c|}
\hline & Number $(\mathrm{n})$ & Percentage (\%) \\
\hline Antifungal & 1 & 1.4 \\
\hline Amoxicillin/clavulanicacid & 1 & 1.4 \\
\hline Ceftriaxone & 3 & 4.1 \\
\hline Ceftazidime & 1 & 1.4 \\
\hline Piperacillin/tazobactam & 32 & 43.8 \\
\hline Meropenem & 11 & 15.1 \\
\hline Vancomycin & 1 & 1.4 \\
\hline Levofloxacin & 2 & 2.7 \\
\hline Cefuroxime & 1 & 1.4 \\
\hline Metronidazole & 1 & 1.4 \\
\hline Meropenem + vancomycin & 1 & 1.4 \\
\hline Piperacillin/tazobactam + fluconazole & 1 & 1.4 \\
\hline Piperacillin/tazobactam + amikacin & 6 & 8.2 \\
\hline Piperacillin/tazobactam + vancomycin & 2 & 2.7 \\
\hline Amoxicillin/clavulanicacid + ciprofloxacin & 1 & 1.4 \\
\hline Amoxicillin/clavulanicacid + azithromycin & 1 & 1.4 \\
\hline Ceftriaxone + azithromycin & 4 & 5.5 \\
\hline N/A & 3 & 4.1 \\
\hline Total & 73 & 100.0 \\
\hline
\end{tabular}


This finding is in accordance with Piukovics et al., who showed a fever prevalence of $34.5 \%$ in a recent retrospective study that analyzed isolates of bacteremias in 469 patients with hematological malignancies from 2005 to 2008 in a Hungarian hospital [10].

In our sample the majority of febrile episodes took place while patients were being treated for their hematology-oncology disease, most notably with intensive protocols. Prophylactic antimicrobials were commonly prescribed, particularly antibiotics and anti-virus; most patients had medical devices. These facts are also in accordance with the most updated literature.

Regarding causes of fever, it is noteworthy that $42.5 \%$ of all febrile episodes were of unknown origin, a concept modified by Engelhart et al. that means "fever without evidence of a specific infection at any site, without isolation of a specific microorganism from body specimens or without any apparent non-infectious cause" [6]. This number is comparable with the findings of Burutaran et al., who found a prevalence of $38 \%$ of fever or unknown origin [9], and with a prevalence of 53\% described by Piukovics et al. [10]. Studies suggest that at least half of all neutropenic patients with fever of unknown origin have an occult infection, underlining the relevance of this entity [6].

Febrile episodes with symptoms, laboratory or other tests positive findings and with microbiological isolates were mainly due to bacteremia (20.5\%) and respiratory tract infections. Burutaran et al., Klastersky et al., Viscoli et al. and Winston et al. reported similar results [5] [7] [9] [11].

Diagnostic yield of microbiological tests, meaning the proportion of isolates in the totality of samples collected, was around $25 \%$. This percentage falls short the 43\% reported by Engelhart et al. [6] or the 59.3\% described by Burutaran et al. [9]. Given the relevance of microbiological results in this context, several studies have addressed strategies to raise sensitivity. A frequently cited approach is to combine the use of enriched media with extended incubation periods, so that fastidious and sensitive microorganisms can grow. However, other authors believe that cultural means cannot develop more and the solution most probably involves molecular techniques such as fluorescence in situ hybridization (FISH) or polymerase chain reaction (PCR) amplification. Both these tools have the potential of accelerating infection diagnosis, but they cannot obviate the need of classical cultures, since they don't provide susceptibility patterns [12]. Serum biomarkers such as procalcitonin have also been studied with the same purpose [13] [14]. Hoeboer et al., for example, showed that procalcitonin is useful to exclude bacteremia in several contexts but its use in this population is limited by the unpredictable effect of neutropenia, immunity dysfunction and prophylactic antimicrobials use on sensitivity and specificity [13].

Concerning microbiological isolates, we found a Gram-negative predominance (two thirds versus one third of Gram-positive). Although microbiological patterns are site-specific, most studies also show a trend towards the predominance of Gram-negative [7]. Burutaran et al., for instance, reported almost $75 \%$ of Gram-negatives [9]. 
However, trends of microbiological isolates have changed throughout time. In the decades of 70 and 80 Gram-negative infections were more prevalent, which was attributed to bacterial translocation through intestinal mucosa. This unimpeded influx of bacteria was secondary to the barrier disruption characterizing severe mucositis, which in turn was secondary to chemotherapy and profound neutropenia [15].

The high severity and mortality of these infections promoted generalization of prophylaxis with antimicrobials, in order to selectively decontaminate gastrointestinal tract of Gram-negative bacteria such as Enterobacteriaceae [15]. Universalization of medical devices implantation took place almost concomitantly. It is reasonable that both actions were behind the transition from a Gram-negative to a Gram-positive preponderance, mainly in bacteremias, that started to be apparent from the 90s on [10] [16]. However, Gram-positive infections weren't as severe as Gram-negative ones. That is the reason why prophylactic antimicrobials were kept unchanged.

From the year 2000 on isolates of Gram-negative were again more prevalent, but displaying different susceptibility patterns: Gram-negative bacteria were now multi-resistant (i.e., resistant to at least three different classes of antibiotics) [8] [17]. Portugal is one of the European countries with the highest percentage of extended-spectrum beta-lactamases producers (as high as 15.5\%) [18]. As such, we believe that prophylactic protocols must be regularly audited by analyzing microbiological isolates and their susceptibility profiles [8] [9] [15].

Randomized placebo-controlled clinical trials showed a decrease in the attack rate and a delay in time of first infection in patients doing prophylactic antimicrobials, but showed no effect on infection incidence or mortality due to infection [16]. In our study we weren't able to calculate with precision which mortality was attributable to infection. However, we assumed that patients dying after a febrile episode died because of infection. We compared outcome at discharge between patients doing and not doing acyclovir, itraconazole or trimethoprim-sulphamethoxazole with a prophylactic purpose. We concluded that there was no statistically significant difference between both groups $(p=0.865)$. A possible explanation is that patients not doing prophylaxis were also patients not being treated for their hematological disease, or doing non-intensive protocols. However, we specifically analyzed patients not doing prophylaxis with antibiotics and we concluded that $81 \%$ were being treated, $48.4 \%$ of which with intensive chemotherapy protocols. We then conclude that antibiotics prophylaxis don't seem to impact infection-related mortality.

Of all febrile cases, $60 \%$ empirically started piperacillin/tazobactam or carbapenems, which is in accordance with the 2010 Infectious Diseases Society of America (IDSA) guidelines [19]. Other antibiotics, such as aminoglycosides, fluoroquinolones and/or vancomycin can be further added to the initial regimen; in our study a combination regimen was empirically chosen in 16 (21.9\%) of cases, six of which by adding an aminoglycoside, vancomycin in three and in two a fluoroquinolone. 
IDSA's guidelines recommend using vancomycin (or other antibacterial withsimilar exclusive action against Gram-positive) in the initial regimen only if there is a high suspicion of catheter-related infection, skin and/or soft tissue infection, pneumonia, or when patient is in shock and there is known endemicity for methicillin-resistant Staphylococcus aureus [19]. In our study three patients had vancomycin in the initial regimen: one because the suspicion was a device-related infection and the other two had chest x-ray documented pneumonia.

IDSA's guidelines also suggest that persistent unexplained fever in patients who are otherwise stable shouldn't lead to an antibiotics change. In addition, if vancomycin (or other antibacterial with similar exclusive action against Gram-positive) had already been started, it should be stopped after two days with no evidence of Gram-positive infection. Conversely, in patients with persistent fever that develop hemodynamic instability it is highly recommended to add antibiotics with the intention of covering resistant Gram-negative and Gram-positive bacteria, anaerobes and fungi. In our study, antibiotics were changed in $41.1 \%$ of cases, either due to persistent fever or to cover microorganisms isolated from blood or urine cultures. $91 \%$ of patients who needed to change antibiotics due to persistent fever were being treated with chemotherapy, $55 \%$ of which with intensive protocols; $50 \%$ had profound neutropenia (absolute neutrophil count, ANC, $<100$ cells $/ \mathrm{mm}^{3}$ ) and $63.5 \%$ were neutropenic.

Concerning antibiotics duration, IDSA's guidelines recommend fine-tuning to the type of infection, patient immunological status and isolated microorganism. For most bacteremias, skin and/or soft tissue infections and pneumonias 10 to 14 days of antibiotics seem to be enough. Exceptions are neutropenic patients, even those with fever of unknown origin, in whom correct antibiotic should be continued until ANC is higher than 500 cells $/ \mathrm{mm}^{3}$. In our study, antibiotics were used, in average, for 11 days. Since the most prevalent foci were bacteremia and respiratory tract infections, duration follows international recommendations.

Infection-related mortality in patients doing intensive chemotherapy protocols is known to be high. Burutaran et al. cite a mortality of $18.5 \%$ in neutropenic patients with fever, value that increases to $35 \%$ when there is isolation of a microorganism in the collected biological products [9].

This suggests that bacteremia is associated with worst prognosis and higher mortality. In this study we compared the outcome at discharge in patients with microbiological isolates with patients with no isolates, and we found no difference $(p=0.279)$. This result might be affected by the small sample size and by the fact that mortality attributable to infection couldn't be calculated with precision, as previously exposed.

This study has several limitations. Firstly, due to its retrospective design it is not possible to infer causal conclusions. Also, the small sample size compromises both the strength of conclusions and the study external validity. Lastly, we chose to analyze only a three-month period of 2015, a decision we took based on practical and time-consuming motives. Engelhart et al. also chose three months in 
different years for their nosocomial infection surveillance study and it seems to be enough to know prevalences and tendencies [6]. The choice of April, September and November was totally random in order to reduce the selection bias, although seasonality issues not specifically addressed might have compromised the true randomness of our results.

\section{Conclusions}

Our retrospective study, analyzing febrile episodes in a hematology-oncology clinic of a portuguese oncology hospital during a three-month period of 2015, concludes that fever is common in our sample. We then hypothesize that fever is common amongst this population.

Although not an aim of this study, we ended up auditing health personnel actions and decisions in a febrile episode. As such, we concluded the team is trained to assume an infection when fever takes place, which translates in automatically collecting biological products (with an obvious preponderance for blood cultures) and initiating empirical antimicrobials as recommended by international guidelines.

However, we also realized that diagnostic yield of biological products cultures is low. This conclusion deserves further reflection, since it has impact on treatment: for instance, in this study the percentage of patients in which antibiotics were changed due to the cultural result is accordingly low.

In febrile episodes where microorganisms were isolated, Gram-negatives prevail. Given the known high prevalence of multi-resistant microorganisms in Portugal, it would be interesting to review all susceptibility results to understand resistant patterns of our hematology-oncology clinic and to adjust both the protocol of antimicrobials prophylaxis and the empirical treatment of infection.

This study was not powered to establish a positive association between infection and mortality in hematology-oncology patients, although this relation is internationally recognized. As such, regular surveillance fever and infection studies, preferably with larger samples, are essential.

\section{Fund}

Publication of this article was sponsored by Liga Portuguesa Contra o Cancro-Núcleo Regional do Norte.

\section{References}

[1] Kluger, M. and Rothenburg, B. (1979) Fever and Reduced Iron: Their Interaction as a Host Defense Response to Bacterial Infection. Science, 203, 374-376. https://doi.org/10.1126/science.760197

[2] Earn, D.J., Andrews, P.W. and Bolker, B.M. (2014) Population-Level Effects of Suppressing Fever. Proceedings of the Royal Society of London B, 281, 1-5. https://doi.org/10.1098/rspb.2013.2570

[3] Evans, S.S., Repasky, E.A. and Fisher, D.T. (2015) Fever and the Thermal Regulation of Immunity: The Immune System Feels the Heat. Nature Reviews Immunolo- 
gy, 15, 335-349. https://doi.org/10.1038/nri3843

[4] McGowan Jr., J.E., Rose, R.C., Jacobs, N.F., Schaberg, D.R. and Haley, R.W. (1987) Fever in Hospitalized Patients. American Journal of Medicine, 82, 580-586. https://doi.org/10.1016/0002-9343(87)90103-3

[5] Klastersky, J. (2004) Management of Fever in Neutropenic Patients with Different Risks of Complications. Clinical Infectious Diseases, 39, S32-S37. https://doi.org/10.1086/383050

[6] Engelhart, S., Glasmacher, A., Exner, M. and Kramer, M.H. (2002) Surveillance for Nosocomial Infections and Fever of Unknown Origin among Adult Hematology-Oncology Patients. Infection Control \& Hospital Epidemiology, 23, 244-248. https://doi.org/10.1086/502043

[7] Mikulska, M., Viscoli, C., Orasch, C., Livermore, D.M. and Averbuch, D. (2014) Aetiology and Resistance in Bacteraemias among Adult and Paediatric Haematology and Cancer Patients. Journal of Infection, 68, 321-331. https://doi.org/10.1016/j.jinf.2013.12.006

[8] Aynioglu, A., Mutlu, B. and Hacihanefioglu, A. (2016) A Comparison of the Efficacy of Piperacillin-Tazobactam and Cefoperazone-Sulbactam Therapies in the Empirical Treatment of Patients with Febrile Neutropenia. Revista Espanola de Quimioterapia, 29, 69-75.

[9] Boada Burutaran, M., Guadagna, R., Grille, S., Stevenazzi, M., Guillermo, C. and Diaz, L. (2014) Results of High-Risk Neutropenia Therapy of Hematology-Oncology Patients in a University Hospital in Uruguay. Revista Brasileira de Hematologia e Hemoterapia, 37, 28-33. https://doi.org/10.1016/j.bjhh.2014.11.012

[10] Piukovics, K., Terhes, G., Lázár, A., Tímár, F., Borbényi, Z. and Urbán, E. (2015) Evaluation of Bloodstream Infections during Chemotherapy-Induced Febrile Neutropenia in Patients with Malignant Hematological Diseases: Single Center Experience. European Journal of Microbiology and Immunology, 5, 199-204. https://doi.org/10.1556/1886.2015.00021

[11] Winston, D.J., Lazarus, H.M., Beveridge, R.A., Hathorn, J.W., Gucalp, R., Ramphal, R., et al. (2001) Randomized, Double-Blind, Multicenter Trial Comparing Clinafloxacin with Imipenem as Empirical Monotherapy for Febrile Granulocytopenic Patients. Clinical Infectious Diseases, 32, 381-390. https://doi.org/10.1086/318500

[12] Peters, R.P.H., Van Agtmael, M.A., Danner, S.A., Savelkoul, P.H.M. and Christina, M.J.E. (2004) New Developments in the Diagnosis of Bloodstream Infections. The Lancet Infectious Diseases, 4, 751-760.

[13] Hoeboer, S.H., Van Der Geest, P.J., Nieboer, D. and Groeneveld, A.B.J. (2015) The Diagnostic Accuracy of Procalcitonin for Bacteraemia: A Systematic Review and Meta-Analysis. Clinical Microbiology and Infection, 21, 474-481.

https://doi.org/10.1016/j.cmi.2014.12.026

[14] Hangai, S., Nannya, Y. and Kurokawa, M. (2015) Role of Procalcitonin and C-Reactive Protein for Discrimination between Tumor Fever and Infection in $\mathrm{Pa}$ tients with Hematological Diseases. Leukemia \& Lymphoma, 56, 910-914. https://doi.org/10.3109/10428194.2014.938329

[15] Hammond, S.P. and Baden, L.R. (2008) Antibiotic Prophylaxis for Patients with Acute Leukemia. Leukemia \& Lymphoma, 49, 183-193. https://doi.org/10.1080/10428190701769673

[16] Baden, L.R. (2005) Prophylactic Antimicrobial Agents and the Importance of Fitness. New England Journal of Medicine, 353, 1052-1054. https://doi.org/10.1056/NEJMe058133 
[17] Gudiol, C., Tubau, F., Calatayud, L., Garcia-Vidal, C., Cisnal, M., Sánchez-Ortega, I., et al. (2011) Bacteraemia Due to Multidrug-Resistant Gram-Negative Bacilli in Cancer Patients: Risk Factors, Antibiotic Therapy and Outcomes. Journal of Antimicrobial Chemotherapy, 66, 657-663. https://doi.org/10.1093/jac/dkq494

[18] Winokur, P.L., Canton, R., Casellas, J.-M. and Legakis, N. (2001) Variations in the Prevalence of Strains Expressing an Extended-Spectrum Beta-Lactamase Phenotype and Characterization of Isolates from Europe, the Americas and the Western Pacific region. Clinical Infectious Diseases, 32, S94-S103. https://doi.org/10.1086/320182

[19] Freifeld, A.G., Bow, E.J., Sepkowitz, K.A., Boeckh, M.J., Ito, J.I., Mullen, C.A., et al. (2011) Clinical Practice Guideline for the Use of Antimicrobial Agents in Neutropenic Patients with Cancer: 2010 Update by the Infectious Diseases Society of America. Clinical Infectious Diseases, 52, e56-e93.

https://doi.org/10.1093/cid/cir073 